\title{
Minireview
}

\section{Immunotherapy approaches on innate immunity for SARS-Cov-2}

\author{
B. A. ARSLAN ${ }^{1}$, A. C. TIMUCIN²
}

\begin{abstract}
${ }^{1}$ Department of Molecular Biology and Genetics, Faculty of Engineering and Natural Sciences, Üsküdar University, Istanbul, Turkey; ${ }^{2}$ Department of Chemical and Biological Engineering, Faculty of Engineering and Natural Sciences, Uskudar University, Uskudar, Istanbul, Turkey
\end{abstract}

Received May 5, 2020; accepted June 15, 2020

\begin{abstract}
Summary.-Severe acute respiratory syndrome coronavirus 2 (SARS-CoV-2) has caused fatal outbreaks of pneumonia. The similarity of S protein of SARS-CoV-2 with SARS-CoV and RaTG13 is about $76 \%$ and $97 \%$, respectively. Also its potential receptor-binding domain (RBD) shows similarity with approximately $74 \%$ and $90.1 \%$ for SARS-CoV and RaTG13, respectively. SARS-CoV-2 has been shown to use the SARS-CoV receptor ACE2 for entry and serine protease TMPRSS2 for S protein priming. Sialic acids are primarily expressed by vertebrates and some microbial pathogens improving the ability to avoid immune system of vertebrate host. Interactions of sialic acid-binding Ig-like lectins (Siglecs) with their ligands play an important role in modulating immune cell function activities as their regulators. Therefore, while Siglecs help immune cells to distinguish between self and non-self, non-self ligands of some sialylated pathogens can recognize Siglecs and reduce immune cell responses or escape from immune surveillance. In this review, innate immunity in SARS-Cov-2 infection was discussed through Siglecs, especially Siglec-7, Siglec-3, NKG2A and neuraminidases.
\end{abstract}

Keywords: SARS-Cov-2; siglecs; NK cells; NKG2A; neuraminidases

\section{Introduction}

Coronaviruses (CoVs) are common human and animal pathogens that can be clasified as zoonoses and cause serious respiratory disease syndromes (Qing et al., 2020). Coronaviruses are enveloped RNA viruses divided into 4 types as $\alpha, \beta, \gamma$ and $\delta$ (Tortorici et al., 2019). Serious acute respiratory syndrome coronavirus (SARS-CoV), SARS-Cov-2 and the Middle East respiratory syndrome

E-mail: belkisatasever.arslan@uskudar.edu.tr; phone: +90-216400-22-22.

Abbreviations: $\mathrm{CoV}(\mathrm{s})=$ coronavirus(es); $\mathrm{NK}=$ natural killer cells; NKG2A = NK group 2 member A; NTD = N-terminal domain; RBD = receptor-binding domain; SARS-CoV-2 = severe acute respiratory syndrome coronavirus 2 ; Siglecs = sialic acidbinding Ig-like lectins coronavirus (MERS-CoV) have caused fatal outbreaks of pneumonia. Other members of the Coronaviridae family of zoonotic origin are endemic in the human population and account for up to $30 \%$ of mild respiratory infections. Among these viruses, the ones from $\alpha$-coronaviruses are $\mathrm{HCoV}-\mathrm{NL} 63$ and HCoV-229E, and HCoV-OC43 and HCoVHKU1 are members of $\beta$-coronaviruses. Currently, there is no specific antiviral therapy or vaccine to combat especially fatal coronaviruses (Hoffmann et al., 2020; Ou et al., 2020; Tortorici et al., 2019).

CoVs can be transmitted from animals to humans and cause serious illness. For this zoonotic conduction, CoVs use spike (S) proteins that bind the virus to host cell receptors. $S$ protein is the main antigen in the virus and induces the production of neutralizing antibodies during infection (Ou et al., 2020; Qing et al., 2020; Tortorici et al., 2019). $S$ proteins also provide virus-cell membrane fusion. 
It has two receptor binding domains, S1 and S2. While S1 binds to sialic acid of the host cell, the other domain S2 recognizes host transmembrane proteins (Ou et al., 2020; Qing et al., 2020; Tortorici et al., 2019; Bertram et al., 2011; Shulla et al., 2011).

Two receptors in host cells bound by $S$ proteins have been identified for human coronaviruses: CD13 for hCoV229E and Angiotensin converting enzyme 2 (ACE2) for SARS-CoV, SARS-Cov-2 and hCoV-NL63 (Ou et al., 2020; Gierer et al., 2013; Kawase et al., 2012). Binding of the S protein to host cell receptors is an important determinant of viral cell and organ tropism (Gierer et al., 2013). S proteins accumulate on the plasma membrane of the infected cell. Sialic acids have been shown to play a role in facilitating CoV infection and CoV cell propagation (Qing et al., 2020).

ACE2 is widely distributed especially in alveolar type II cells (AT2) and capillary endothelium. Although ACE2 expression in lungs has been demonstrated for both type I and type II pneumocytes, SARS-CoV cell tropism is not fully associated with ACE2 expression. It is suggested that other factors are needed to explain the pathogenesis of this disease. $S$ proteins are synthesized as inactive precursors and become active on proteolytic cleavage by proteases (Gierer et al., 2013; Matsuyama et al., 2010).

$S$ protein of CoVs can be activated by cathepsins, furin, trypsin, elastase, hypoxanthine-aminopterin-thymidine (HAT), transmembrane protease serine protease-2 (TMPRSS2), TMPRSS11a, and TMPRSS-4 proteases. TMPRSS2 is expressed in epithelial cells of human lungs (Ou et al., 2020; Gierer et al., 2013; Kawase et al., 2012; Bertram et al.,2011). Entering of the virus into the cell through cell membrane depends on the presence of these proteases in the cell (Ou et al., 2020). Endosomal cathepsin B and especially cathepsin $\mathrm{L}$ are required for entering of SARSCoV and hCoV-229E into the cell by endocytosis (Gierer et al., 2013; Bertram et al., 2012; Kawase et al., 2012; Glowacka et al., 2011; Matsuyama et al., 2010). There are several proteases that can work as SARS-CoV entry cofactors, including cathepsin L, elastase, trypsin, factor Xa, and plasmin. Without ACE2 binding, these soluble proteases excessively separate and neutralize virus spikes (Shulla et al., 2011). Understanding of functions of these proteases in SARSCov-2 can contribute to elucidation of possible functional differences between SARS-Cov and SARS-Cov-2.

TMPRSS2 activates SARS-CoV S protein on the cell surface following receptor binding during viral entry into cells (Kawase et al., 2012). TMPRSS2 affects virus entry, but not other stages of virus replication (Matsuyama et al., 2010). Neutralizing of TMPRSS2 with antibodies can reduce viral recognition. ACE2 and TMPRSS2 are coexpressed in type II pneumocytes. TMPRSS2 distribution correlates with SARS-CoV infection in lungs (Bertram et al., 2012; Glowacka et al., 2011; Matsuyama et al., 2010).

\section{SARS-CoV-2}

The similarity of S protein of SARS-CoV-2 with SARSCoV and RaTG13 is about $76 \%$ and $97 \%$, respectively. Also its potential receptor-binding domain (RBD) shows similarity with approximately $74 \%$ and $90.1 \%$ for SARS-CoV and RaTG13, respectively. SARS-CoV-2 has been shown to use the SARS-CoV receptor ACE2 for entry and serine protease TMPRSS2 for S protein priming (Hoffmann et al., 2020; Leng et al.,2020; Ou et al., 2020). SARS-CoV-2 has been identified as the genus $\beta$-coronavirus and shares $79.5 \%$ sequence homology with SARS-CoV (Zheng et al., 2020a).

Lymphocytes play an important role in maintaining immune system function. Therefore, there is a relationship between changes in lymphocyte numbers and subgroups and viral pathogenic mechanisms. In patients infected with SARS-CoV- $2, \mathrm{CD}^{+}{ }^{+} \mathrm{T}$ cells, $\mathrm{CD} 8^{+} \mathrm{T}$ cells, $\mathrm{B}$ cells, and natural killer (NK) cells have been shown to decrease in severe cases to a lower level than mild cases (Wang et al., 2020).

Another study found that the total number of NK and CD8 ${ }^{+} \mathrm{T}$ cells decreased significantly in patients with SARS-CoV-2 infection and increased expression of NKG2A, an inhibitory receptor in these cells. It is thought that downregulation of NKG2A expression may be associated with disease control in SARS-CoV-2 patients (Zheng et al., 2020a). The virus is able to induce cytokine storm in the lungs, overproducing cytokines such as IL-2, IL-6, IL-7, GSCF. Therefore, avoiding cytokine storm may be the key to treating SARS-CoV-2 infected patients (Leng et al., 2020). The fact that NKG2A is expressed in both NK cells and CD8 ${ }^{+}$ $T$ cells makes it an important treatment target against virus infections. Also, can the HLA-E expression levels and polymorphism studies provide information regarding sensitivity to SARS-Cov-2 and progression of the disease?

When we separate treatment processes of the infection as early and late phase, the first part can be effective solution for low NK activity and immunotherapy approaches that will increase innate immunity, and in the late phase, inhibitory cytokine treatment approaches that will suppress cytokine storm. Can the factor lowering NK activity be explained by innate immunity-escape mechanism of SARS-Cov-2? In this review, this possibility is discussed through the role of sialic acids in innate immunity. To determine the main regulatory factors, increasing detailed proteomics studies and biomarkers showing progression of the disease will contribute to the identification of possible drug targets.

\section{Sialic acids}

Sialic acids are made up of over 50 carbohydrate families that share a nine-carbon backbone. Sialic acid 
(Neu5Ac) can attach to proteins and lipids as terminal sugars of oligosaccharides (C1-9) (Mikulak et al., 2017).

They are primarily expressed by vertebrates and some microbial pathogens improving the ability to avoid immune system of vertebrate host. These negatively charged sugars are found at the terminal of a number of glycoconjugates, including $\mathrm{N}$-linked glycan chains, O-linked mucin chains, and glycosphingolipids on proteins (Pillai et al., 2012).

In humans there is Neu5Ac instead of Neu5Gc which is commonly found in other mammals. However, the dietary consumption of Neu5Gc, primarily from red meat, can be metabolically incorporated into cellular glycoproteins and glycolipids, following the same route used by the endogenous sialic acid Neu5Ac after absorption by the gastrointestinal tract. The exogenous Neu5Gc consumed in this way is presented in the cell surface glycocalyx where it can act as a foreign "xeno-autoantigen". Although the amounts included are small, most people have circulating anti-Neu5Gc "xeno-autoantibodies" and therefore Neu5Gc can develop local chronic inflammation in the accumulation sites (Kawanishi et al., 2019).

Sialic acids are host recognition elements binding hemagglutinins or spike proteins of type A and B influenza viruses. These hemagglutinins represent viral sialic acid-binding lectins. Hemagglutinin or hemagglutinin esterases of type $\mathrm{C}$ influenza viruses and some nidoviruses may recognize the 9-O-acetylated form of sialic acid. This form of sialic acid is necessary for the virus to enter the human cell. Sialic acid glyco-epitope is sensitive to hemagglutinin esterase sialic acid O-acetyl esterase activity of bovine coronavirus (Mahajan et al., 2019; Pillai et al., 2012).

Sialic acid can regulate the alternative way of complement activation. Major serum protein complement factor $\mathrm{H}$ binds to the exocyclic side chain of sialic acids on host cells and prevents the interaction of complement factor $\mathrm{B}$ with $\mathrm{C} 3 \mathrm{~b}$ and $\mathrm{C} 3$ convertase formation. Many microbial pathogens can escape natural immunity in vertebrates by binding to factor $\mathrm{H}$ or by triggering sialic acid-binding receptors in innate immune cells. Selectins, C type or calcium dependent lectins have sialic acid containing ligands (Kim et al., 2017; Pillai et al., 2012).

\section{Siglecs}

Siglecs (sialic acid-binding Ig-like lectins) are characterized by the Ig domain, which mediates binding of sialic acid (Crocker et al., 2001).

Siglecs can be divided into 2 groups: first group; Siglec-1 (Sialoadhesin), Siglec-2 (CD22), Siglec-4 (MAG) and Siglec-15. The second group is CD33-related Siglecs. Members of this group have two to five Ig-like domains that share $50 \%$ to $80 \%$ sequence identity (Lübbers et al., 2018; Macauley et al., 2014; Nicoll et al., 2003). Siglec-12 has lost the ability to bind sialic acids (Mikulak et al., 2017).

Interactions of Siglecs with their ligands play an important role in modulating immune cell functions activities as their regulators. Therefore, while Siglecs help immune cells to distinguish between self and non-self, non-self ligands of some sialylated pathogens can recognize Siglecs and reduce immune cell responses or escape from immune surveillance (Macauley et al., 2014).

Siglecs are mostly derived from hematopoietic lineage and are structurally expressed on cells of natural immunity such as monocytes/macrophages, neutrophils, basophils, eosinophils, dendritic cells (DCs) and natural killer (NK) cells. Siglec-1 and -11 are found structurally only in macrophages. Siglec-5 are expressed on B cells and neutrophils (Mikulak et al., 2017). Siglecs generally lead to a strong inhibitory signal when they bind to sialic acids. Pathogens increase sialic acid expression as a mechanism that modifies the immune system (Lübbers et al., 2018; Kim et al., 2017; Mikulak et al., 2017).

Macrophages localized in the marginal zone of the spleen and in the subcapsular sinuses of the lymph nodes express a high level of CD169. CD169 is also known as a sialoadhesin or Ig-like lectin-1 (Siglec-1) that binds sialic acid. Unlike other Siglecs, sialoadhesin have 17 Iglike domains. $\mathrm{CD}^{169}{ }^{+}$macrophages play a major role in inducing both humoral and cellular adaptive immunity. $\mathrm{CD}_{169}{ }^{+}$macrophages can directly stimulate $\mathrm{CD} 8^{+} \mathrm{T}$ cells after viral infection (van Dinther et al., 2018; Crocker et al., 2001). Siglec-1 and Siglec-2 mediate cell-to-cell interaction through sialylated glycoconjugates. Masking of Siglecs on the cell surface as a result of cis interaction with sialic acid prevents cell-cell interactions (Crocker et al., 2001).

Treatment of monocytes with Siglec-3 Ab increases their IL-8, TNF- $\alpha$ and IL-1beta proinflammatory cytokine production (Lübbers et al., 2018). Considering these results, can Siglec-3 activation suppress cytokine production in the late phase when cytokine storm is established in SARS-Cov-2-infected patients?

Siglec-7 (also called p75/AIRM1) is a sialic acid-binding receptor expressed on NK cells and monocytes (Nicoll et al., 2003). NK cells express endogenous ligands for Siglec-7, which could bind Siglec-7 and possibly keep these cells in an inhibited state (Pillai et al., 2012). In terms of the amino acid sequence, Siglec-7 and Siglec-9 are approximately in $80 \%$ similar to each other (Daly et al., 2019). Siglec-7 are structurally masked on NK cells. However, when it is treated with sialidase, Siglec-7 can interact with GD3 and inhibit killing. Expression of GD3 on target cells has been shown to increase susceptibility to killing independently of Siglec-7. It can modulate NK cell killing activity and has 

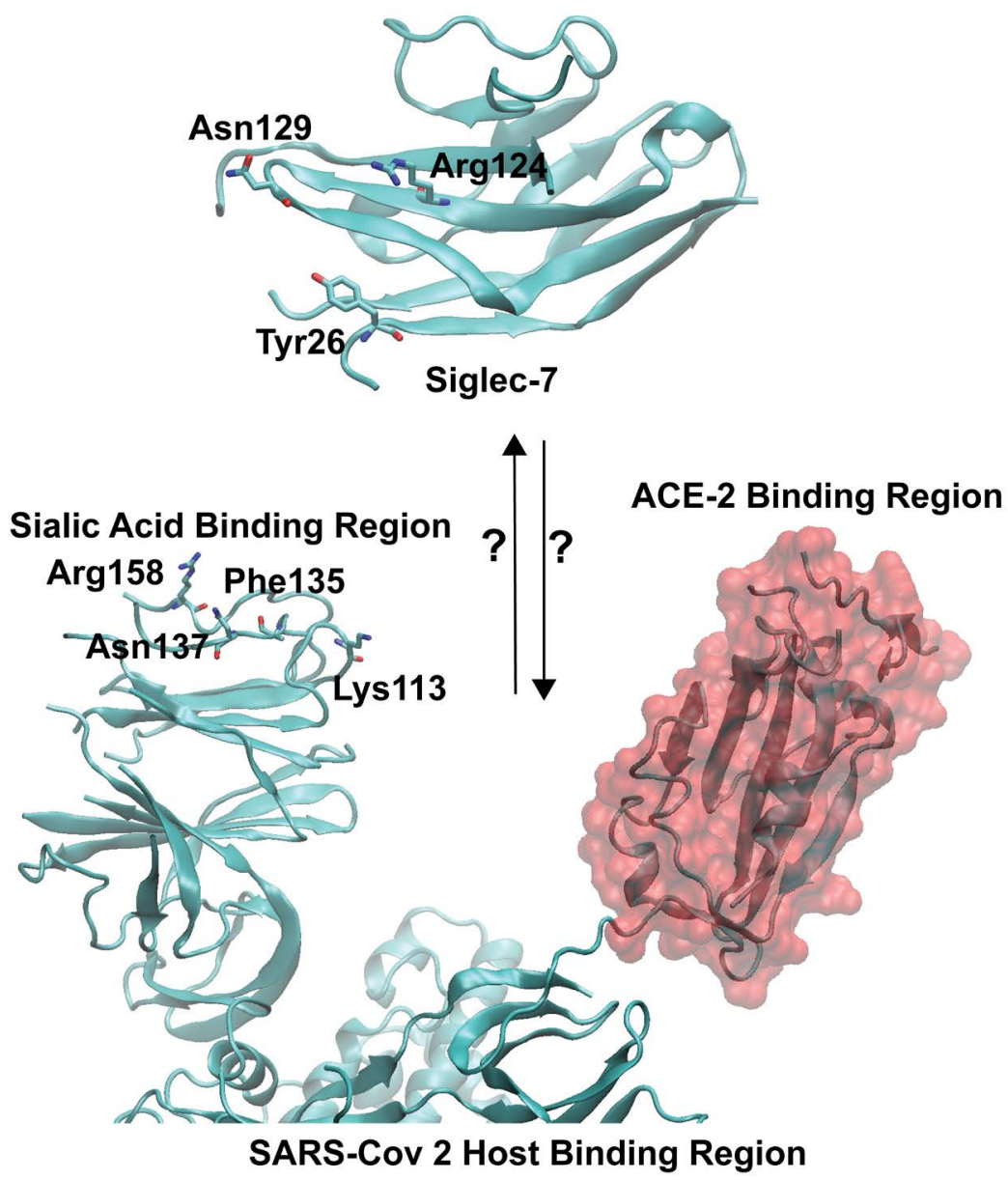

Fig. 1

Spike protein of SARS-Cov-2 has its own residues as potential targets for Siglec-7

Spike protein has its own sialic acid recognition region for host cells targeting, together with ACE-2 binding region. Do these two mechanisms interact under certains settings? The answer to this question has yet to be determined. Siglec-7 (PDB ID:2DF3), SARS-Cov-2 spike protein (PDB ID:6VSB). The amino acids, responsible for binding to sialic acid are shown. ACE-2 binding region on spike protein is highlighted with surface representation together with secondary structure representation.

significant effects on tumor escape from NK cell cytotoxicity (Nicoll et al., 2003).

Siglec-7, which is the most abundantly found Siglec on natural killer cells, presents high binding preference towards $\alpha(2,8)$-linked disialic acids, lower preference toward $\alpha(2,6)$ - and lowest preference for $\alpha(2,3)$-linked sialic acids (Alphey et al., 2003; Zheng et al., 2020b). Within the frame of this property of Siglec-7, it has also been shown to be involved in viral infections. Low expression levels of Siglec-7 on NK cell populations was shown to be associated with viremia of HIV (Brunetta et al., 2009). Further in vitro studies revealed that sialic acid residues on HIV gp120 utilizes Siglec-7 for protection against immune system and systemic increase of soluble Siglec-7 during HIV infections was suggested to be due to NK cell apoptosis (Brunetta et al., 2009; Mikulak et al., 2017; Varchetta et al., 2013). Similar Siglec-7 dependent events were also observed for HCV and HBV infections (Zheng et al., 2020b). Given that spike protein of SARS-Cov-2 contains glycosylated regions (Walls et al.,2020), here we speculate that Siglec-7, probably together with other Siglecs, may contribute to the escape of SARS-Cov-2 from immunological surveillance, which prompts the need for further studies in this area. On the other hand, a potential drug candidate in COVID-19, chloroquine, has been shown to inhibit the sialic acid-binding of NTD region of the spike protein, potentially leading to decreased binding of the virus to the sialic acid residues found on the host cells (Fantini et al., 2020). If exists, could Siglec-7 or any other Siglec interaction with glycosylated viral amino acid residues enhance or disrupt the capac- 
ity of the virus to interact with host sialic acid residues though its NTD region? Discovery of such interactions will pave a path to design and development of new biomimicry based inhibitors and drugs (Fig. 1).

Enveloped viruses, such as HIV and porcine reproductive and respiratory syndrome virus (PRRSV), are covered with sialic acids that can be recognized by sialoadhesin in macrophages and promote uptake and infection. Sialoadhesin has been shown to be the major receptor for GM3 providing HIV trans-infection and spread to endosomal compartments to ensure and spread. HIV can interact with other Siglecs, including Siglec-3, Siglec-5, Siglec-7 and Siglec-9 via glycoprotein 120 (gp120) (Macauley et al., 2014).

A soluble form of Siglec-7 has been shown to act as a biomarker in HIV-1 infected patients. It has been shown that Siglec-7 level is increased in the supernatant of NK cells stimulated in vitro with $\mathrm{HCV}$ and there is a decrease in Siglec-7 expression from NK cell surface (Mikulak et al., 2017). It has been hypothesized that soluble Siglec-7 may cause spreading of the infection and increase the susceptibility to infection due to its HIV-1 carrying capacity. (Mikulak et al., 2017).

Due to the very limited expression of the Siglecs in the immune system and the differential expression of the Siglecs in various immune cells, they are increasingly recognized as targets for the development of immunotherapeutics (Mikulak et al., 2017; Macauley et al., 2014). Siglec binding sites are masked on the same cell surface in cis form by interacting with high and low affinity sialic acids. It can interact in trans form when it encounters a high affinity ligand or pathogen that can compete with this interaction (Mikulak et al., 2017).

\section{Natural killer (NK) cells}

The immune response against viral infection is based on effector mechanisms from both a natural and adaptive immunity. Among these mechanisms, cytotoxicity mediated by NK cells and cytotoxic CD8 ${ }^{+} \mathrm{T}$ cells (CTL) is responsible for killing infected cells. In human T lymphotropic virus type 1 (HTLV-1) infection, while NK cells try to limit proliferation of virus-infected cells and the proviral load in the early stages of infection, CTLs are responsible for controlling viral delay (Bangham, 2009).

NK cells play a central role in defending against viral infections and eliminating transformed cells. Its activity is regulated by the interaction of activating or inhibitory NK cell surface receptors, effects of cytokines and their interactions with other immune cells. Most inhibitor receptors recognize the major histocompatibility complex (MHC) class I and provide tolerance of NK cells against self-antigens (Jarahian et al., 2009). Activating receptors on NK cells include CD16; NKG2D; natural cytotoxicity receptors (NCR) NKp30, NKp44 and NKp46 (AtaseverArslan et al., 2013; Jarahian et al., 2009).

Hemagglutinin (HA) of the influenza virus and hemagglutinin-neuraminidase $(\mathrm{HN})$ proteins of Sendai virus can bind to NKp46 and NKp44 and activate NK cells. Binding of NKp44 and NKp46 to viral HA mediated by specific sialic acid residues found in these receptors leads to the elimination of infected cells (Duev-Cohen et al., 2016). NKp30 do not bind the HA of influenza virus. The human cytomegalovirus pp65 protein has been shown to bind NKp30 and inhibit its function. Also, HIV virus and herpes simplex virus have been shown to upregulate expression of NCR ligands in infected cells (Bar-On et al., 2014; Jarahian et al., 2009). Influenza virus has been shown to use viral neuraminidase (NA) protein to avoid NKp46-mediated elimination, and NA inhibition leads to increased elimination of influenza virus-infected cells in vitro and in vivo (Bar-On et al., 2014).

There are four types of sialidase as neuraminidases (NEU) 1-4. They function by separating sialic acids from glycans by hydrolysis. Increased Siglec-7 and Siglec- 9 levels in NK cells of healthy donors treated with sialidase neuraminidase have been observed. Treating only NK cells with sialidase is thought to prevent masking of Siglec-7 with sialic acids in cis form and may increase NK cytotoxicity. Another potential therapeutic approach is using of Siglec-7 inhibitors to prevent Siglec-7-mediated NK activity inhibition (Daly et al., 2019).

Consequently, understanding of importance of Siglec-7 and neuraminidase therapy for SARS-Cov-2 infection may contribute to enhancing innate immunity in the early phase of infection. Increasing NKG2A level in patients infected with SARS-Cov-2 is an important finding related to innate immunity escape mechanisms. Considering this result, investigating HLA-E levels and polymorphisms in patients may contribute to the determination of new drug targets. Could increasing the activity of inhibitory mechanisms in cytokine producing cells such as Siglec-3 for cytokine storm be an effective treatment and approach for the late phase of infection?

\section{References}

Alphey, MS, Attrill H, Crocker PR, van Aalten DM (2003): High resolution crystal structures of Siglec-7. Insights into ligand specificity in the Siglec family. J. Biol. Chem. 278, 3372-3377.

Atasever Arslan B, Erdem-Kuruca S, Karakas Z, Erman B, Ergen A (2013): Effects of micro environmental factors on natural killer activity (NK) of beta thalassemia major patients. Cell. Immunol. 282, 93-99. https://doi. org/10.1016/j.cellimm.2013.04.012 
Bangham CR (2009): CTL quality and the control of human retroviral infections. Eur. J. Immunol. 39, 1700-1712. https://doi.org/10.1002/eji.200939451

Bar-On Y, Seidel E, Tsukerman P, Mandelboim M, Mandelboim O (2014): Influenza virus uses its neuraminidase protein to evade the recognition of two activating $\mathrm{NK}$ cell receptors. J. Infect. Dis. 210, 410-418. https://doi. org/10.1093/infdis/jiu094

Bertram S, Glowacka I, Müller MA, Lavender H, Gnirss K, Nehlmeier I, Niemeyer D, He Y, Simmons G, Drosten C, Soilleux EJ, Jahn O, Steffen I, Pöhlmann S (2011): Cleavage and activation of the severe acute respiratory syndrome coronavirus spike protein by human airway trypsin-like protease. J. Virol. 85, 13363-13372. https:// doi.org/10.1128/JVI.05300-11

Bertram S, Heurich A, Lavender H, Gierer S, Danisch S, Perin P, Lucas JM, Nelson PS, Pöhlmann S, Soilleux EJ (2012): Influenza and SARS-coronavirus activating proteases TMPRSS2 and HAT are expressed at multiple sites in human respiratory and gastrointestinal tracts. PLoS One 7, e35876. https://doi.org/10.1371/journal. pone.0035876

Brunetta E, Fogli M, Varchetta S, Bozzo L, Hudspeth KL, Marcenaro E, Moretta A, Mavilio D (2009): The decreased expression of Siglec-7 represents an early marker of dysfunctional natural killer-cell subsets associated with high levels of HIV-1 viremia. Blood 114, 3822-3830. https://doi.org/10.1182/blood-2009-06-226332

Crocker PR, Varki A (2001): Siglecs in the immune system. Immunology 103,137-145. https://doi.org/10.1046/i.00192805.2001.01241.x

Daly J, Carlsten M, O'Dwyer M (2019): Sugar free: Novel immunotherapeutic approaches targeting Siglecs and sialic acids to enhance natural killer cell cytotoxicity against cancer. Front. Immunol. 10, 1047. https://doi. org/10.3389/fimmu.2019.01047

Duev-Cohen A, Bar-On Y, Glasner A, Berhani O, Ophir Y, LeviSchaffer F, Mandelboim M, Mandelboim O (2016): The human 2B4 and NTB-A receptors bind the influenza viral hemagglutinin and co-stimulate NK cell cytotoxicity. Oncotarget 7, 13093-13105. https://doi. org/10.18632/oncotarget.7597

Fantini J, Di Scala C, Chahinian H, Yahi N (2020): Structural and molecular modelling studies reveal a new mechanism of action of chloroquine and hydroxychloroquine against SARS-CoV-2 infection. Int. J. Antimicrob. Agents 105960. https://doi.org/10.1016/j.ijantimicag.2020.105960

Gierer S, Bertram S, Kaup F, Wrensch F, Heurich A, Krämer-Kühl A, Welsch K, Winkler M, Meyer B, Drosten C, Dittmer U, von Hahn T, Simmons G, Hofmann H, Pöhlmann S (2013): The spike protein of the emerging betacoronavirus EMC uses a novel coronavirus receptor for entry, can be activated by TMPRSS2, and is targeted by neutralizing antibodies. J. Virol. 87,5502-5511. https://doi. org/10.1128/JVI.00128-13

Glowacka I, Bertram S, Müller MA, Allen P, Soilleux E, Pfefferle S, Steffen I, Tsegaye TS, He Y, Gnirss K, Niemeyer D,
Schneider H, Drosten C, Pöhlmann S (2011): Evidence that TMPRSS2 activates the severe acute respiratory syndrome coronavirus spike protein for membrane fusion and reduces viral control by the humoral immune response. J. Virol. 85, 4122-4134. https://doi. org/10.1128/JVI.02232-10

Hoffmann M, Kleine-Weber H, Schroeder S, Krüger N, Herrler T, Erichsen S, Schiergens TS, Herrler G, Wu NH, Nitsche A, Müller MA, Drosten C, Pöhlmann S (2020): SARS-CoV-2 Cell entry depends on ACE2 and TMPRSS2 and is blocked by a clinically proven protease inhibitor. Cell 181,271-280.e8. https://doi.org/10.1016/j. cell.2020.02.052

Jarahian M, Watzl C, Fournier P, Arnold A, Djandji D, Zahedi S, Cerwenka A, Paschen A, Schirrmacher V, Momburg F (2009): Activation of natural killer cells by newcastle disease virus hemagglutinin-neuraminidase. J. Virol. 83, 8108-8121. https://doi.org/10.1128/JVI.00211-09

Kawanishi K, Dhar C, Do R, Varki N, Gordts PLSM, Varki A (2019): Human species-specific loss of CMP-N-acetylneuraminic acid hydroxylase enhances atherosclerosis via intrinsic and extrinsic mechanisms. Proc. Natl. Acad. Sci. USA 116, 16036-16045. https://doi.org/10.1073/ pnas.1902902116

Kawase M, Shirato K, van der Hoek L, Taguchi F, Matsuyama S (2012): Simultaneous treatment of human bronchial epithelial cells with serine and cysteine protease inhibitors prevents severe acute respiratory syndrome coronavirus entry. J. Virol. 86, 6537-6545. https://doi. org/10.1128/JVI.00094-12

Kim YH, Min KH, Wang Z, Kim J, Jacobson O, Huang P, Zhu G, Liu Y, Yung B, Niu G, Chen X (2017): Development of sialic acid-coated nanoparticles for targeting cancer and efficient evasion of the immune system. Theranostics 7 , 962-973. https://doi.org/10.7150/thno.19061

Leng Z, Zhu R, Hou W, Feng Y, Yang Y, Han Q, Shan G, Meng F, Du D, Wang S, Fan J, Wang W, Deng L, Shi H, Li H, Hu Z, Zhang F, Gao J, Liu H, Li X, Zhao Y, Yin K, He X, Gao Z, Wang Y, Yang B, Jin R, Stambler I, Lim LW, Su H, Moskalev A, Cano A, Chakrabarti S, Min KJ, Ellison-Hughes G, Caruso C, Jin K, Zhao RC (2020): Transplantation of ACE2- mesenchymal stem cells improves the outcome of patients with COVID-19 pneumonia. Aging Dis. 11, 216-228. https://doi.org/10.14336/AD.2020.0228

Lübbers J, Rodríguez E, van Kooyk Y (2018): Modulation of immune tolerance via Siglec-sialic acid interactions. Front. Immunol. 9, 2807. https://doi.org/10.3389/ fimmu.2018.02807

Macauley MS, Crocker PR, Paulson JC (2014): Siglec-mediated regulation of immune cell function in disease. Nat. Rev. Immunol. 14, 653-666. https://doi.org/10.1038/ nri3737

Mahajan VS, Alsufyani F, Mattoo H, Rosenberg I, Pillai S (2019): Alterations in sialic-acid O-acetylation glycoforms during murine erythrocyte development. Glycobiology 29, 222-228. https://doi.org/10.1093/glycob/cwy110

Matsuyama S, Nagata N, Shirato K, Kawase M, Takeda M, Taguchi $\mathrm{F}$ (2010): Efficient activation of the severe acute 
respiratory syndrome coronavirus spike protein by the transmembrane protease TMPRSS2. J. Virol. 84, 12658-12664. https://doi.org/10.1128/JVI.01542-10

Mikulak J, Di Vito C, Zaghi E, Mavilio D (2017): Host immune responses in HIV-1 infection: The emerging pathogenic role of Siglecs and their clinical correlates. Front. Immunol. 23, 314. https://doi.org/10.3389/ fimmu.2017.00314

Nicoll G, Avril T, Lock K, Furukawa K, Bovin N, Crocker PR (2003): Ganglioside GD3 expression on target cells can modulate NK cell cytotoxicity via siglec-7-dependent and -independent mechanisms. Eur. J. Immunol. 33, 1642-1648. https://doi.org/10.1002/eji.200323693

Ou X, Liu Y, Lei X, Li P, Mi D, Ren L, Guo L, Guo R, Chen T, Hu J, Xiang Z, Mu Z, Chen X, Chen J, Hu K, Jin Q, Wang J, Qian Z (2020): Characterization of spike glycoprotein of SARS-CoV-2 on virus entry and its immune crossreactivity with SARS-CoV. Nat. Commun. 11, 1620. https://doi.org/10.1038/s41467-020-15562-9

Pillai S, Netravali IA, Cariappa A, Mattoo H (2012): Siglecs and immune regulation. Annu. Rev. Immunol.30,357-392. https://doi.org/10.1146/annurev-immunol-020711-075018

Qing E, Hantak M, Perlman S, Gallagher T (2020): Distinct Roles for Sialoside and Protein Receptors in Coronavirus Infection.mBio 11, pii: e02764-19. https://doi.org/10.1128/ mBio.02764-19

Saludes JP, Ames JB, Gervay-Hague J (2009): Synthesis and structural characterization of sialic acid-glutamic acid hybrid foldamers as conformational surrogates of alpha-2,8-linked polysialic acid. J. Am. Chem. Soc. 131, 5495-5505. https://doi.org/10.1021/ja808286x

Shulla A, Heald-Sargent T, Subramanya G, Zhao J, Perlman S, Gallagher T (2011): A transmembrane serine protease is linked to the severe acute respiratory syndrome coronavirus receptor and activates virus entry. J. Virol. 85, 873-882. https://doi.org/10.1128/JVI.02062-10
Tortorici MA, Walls AC, Lang Y, Wang C, Li Z, Koerhuis D, Boons GJ, Bosch BJ, Rey FA, de Groot RJ, Veesler D (2019): Structural basis for human coronavirus attachment to sialic acid receptors. Nat. Struct. Mol. Biol. 26, 481-489. https://doi.org/10.1038/s41594-019-0233-y

van Dinther D, Veninga H, Revet M, Hoogterp L, Olesek K, Grabowska J, Borg EGF, Kalay H, van Kooyk Y, den Haan $\operatorname{JMM}(2018)$ : Comparison of protein and peptide targeting for the development of a CD169-based vaccination strategy against melanoma. Front. Immunol. 6, 1997. https://doi.org/10.3389/fimmu.2018.01997

Varchetta S, Lusso P, Hudspeth K, Mikulak J, Mele D, Paolucci S, Cimbro R, Malnati M, Riva A, Maserati R, Mondelli MU, Mavilio D (2013): Sialic acid-binding Ig-like lectin-7 interacts with HIV-1 gp120 and facilitates infection of CD4pos T cells and macrophages. Retrovirology 10,154. https://doi.org/10.1186/1742-4690-10-154

Walls AC, Park YJ, Tortorici MA, Wall A, McGuire AT, Veesler D (2020): Structure, function, and antigenicity of the SARS-CoV-2 spike glycoprotein. Cell 181, 281-292 e6. https://doi.org/10.1016/j.cell.2020.02.058

Wang F, Nie J, Wang H, Zhao Q, Xiong Y, Deng L, Song S, Ma Z, Mo P, Zhang Y. (2020): Characteristics of peripheral lymphocyte subset alteration in COVID-19 pneumonia. J. Infect. Dis. pii: jiaa150. https://doi.org/10.1093/ infdis/jiaa150

Zheng M, Gao Y, Wang G, Song G, Liu S, Sun D, Xu Y, Tian Z (2020a): Functional exhaustion of antiviral lymphocytes in COVID-19 patients. Cell. Mol.Immunol.https:// doi.org/10.1038/s41423-020-0402-2

Zheng Y, Ma X, Su D, Zhang Y, Yu L, Jiang F, Zhou X, Feng Y, Ma F (2020b): The roles of Siglec-7 and Siglec-9 on natural killer cells in virus infection and tumour progression. J. Immunol. Res. 6243819. https://doi. org/10.1155/2020/6243819 